\title{
Le jury criminel en procès. Les opinions doctrinales des auteurs français (1750-1830).
}

\author{
The Criminal Jury at Trial. The Doctrinal Opinions \\ of French Authors (1750-1830)
}

Sylvain Soleil ${ }^{\mathbf{1}}$

Université Rennes 1, France

sylvain.soleil@univ-rennes1.fr

http://orcid.org/0000-0001-7800-3621

À la mémoire de Marie-Yvonne Crépin († 2020)

À l'amie, à l'historienne du droit criminel

Resumo: Le modèle anglais de jury criminel est admiré et réclamé dans l'Europe des Lumières, spécialement en France. Adopté dans l'enthousiasme par les révolutionnaires (1790-1791), il devient l'objet de critiques très vives parce que la pratique des années 1792-1800 révèle des failles dans le système. Les Thermidoriens, puis les membres du Directoire cherchent à rectifier le fonctionnement du double jury d'accusation et de jugement. En vain. Une première controverse doctrinale se déchaîne dans les années 1798-1808, lorsque Napoléon Bonaparte s'interroge sur le maintien du jury. Une seconde controverse doctrinale a lieu après la restauration du roi Louis XVIII, dans les années 1818-1828, lorsqu'il s'agit de réhabiliter le jury anglais comme symbole d'une justice criminelle équilibrée, mais aussi d'aller sur place pour observer en Angleterre la façon dont fonctionne la justice.

Palavras-Chave: Jury criminel; Doctrines; Angleterre; Lumières; Révolution française; Napoléon.

1 Sylvain Soleil est professeur à l'Université de Rennes 1, directeur de l'axe Théorie et Histoire des Systèmes Juridiques de l'IODE (UMR CNRS 6262). Il s'intéresse à l'histoire comparée du droit et s'apprête à publier, aux éditions de la Société de Législation Comparée : Aux origines de l'opposition entre systèmes de common law et de droit codifié. Les controverses anglo-américaines des années 1820-1835. 
ABSTRACT: The English model of the criminal jury was admired in the Europe of the Enlightenment, especially in France. Enthusiastically adopted by the revolutionaries (1790-1791), it became the object of very strong criticism because of the flawed practice of the system in the years 1792-1800. The Thermidoriens, then the members of the Directoire sought to rectify the operating of the double jury of accusation and judgment. In vain. A first doctrinal controversy erupted in the years 1798-1808, when Napoleon Bonaparte questioned whether the jury should be maintained or not. A second doctrinal controversy arose after the restoration of King Louis XVIII, in the years 1818-1828, when it was a question of rehabilitating the English jury as a symbol of balanced criminal justice, but also of going to England to observe how justice worked.

Kerwords: Criminal Jury; Doctrine; England; Enlightenment; French Revolution; Napoleon.

SumÁRıo: Introduction; 1 . Les Lumières et l'engouement pour le jury à l'anglaise; 2 . Les Révolutionnaires et l'adoption du jury à l'anglaise; 3. La pratique judiciaire et le problème des acquittements scandaleux; 4. Le Consulat et la première controverse doctrinale sur le jury; 5. L'Empire, la suppression du jury d'accusation et le maintien du jury criminel; 6. La Restauration et la seconde controverse doctrinale sur le jury à l'anglaise; Conclusions.

\section{INTRODUCTION}

Dans son célèbre ouvrage consacré à La loi de l'imitation, le Français Gabriel Tarde affirme que le développement des sociétés humaines dépend plus d'un processus d'imitation que d'une loi de l'évolution qui imposerait à tous les peuples le même chemin et le même but, par les mêmes stades de développement. Cette loi de l'imitation, explique-t-il, conduit les peuples, dès qu'ils ont relativisé la valeur de la tradition, à s'inspirer de ce qui a été inventé ailleurs (une stratégie militaire, une belle architecture, un procédé commercial, etc.), quand cela paraît plus efficace, plus raffiné ou plus utile. Tarde a été substitut 
du procureur puis juge d'instruction, avant de devenir sociologue. Aussi alimente-t-il sa théorie grâce à des éléments juridiques ${ }^{2}$ :

«On est stupéfait de voir avec quelle rapidité, à certaines époques, se sont répandues des procédures criminelles aussi odieuses qu'absurdes, telles que la torture, ou aussi inefficaces qu'inintelligentes, telles que le jury. La torture a été à la mode en Europe à partir de l'exhumation bolonaise du Droit romain ; et jusqu'au XVI e siècle, elle s'étend comme une inondation sanglante. Au XVIII siècle, c'est du jury qu'on s'éprend partout, sans le connaitre, sur la foi de quelques anglomanes; si bien que tous les cahiers des États généraux en 1789 étaient unanimes sur ce point, comme sur tant d'autres. »

Cette affirmation est intéressante : Tarde, en tant que sociologue, prend acte de la diffusion du jury dans le monde ; Tarde, en tant que juriste, est exaspéré par le succès de cette institution « aussi inefficace qu'inintelligente ». De fait, au moment où il publie son ouvrage (1890), Tarde traduit les sentiments d'une partie des juristes français et européens qui se déclarent radicalement opposés au système des jurés criminels.

De façon simple, ce système confie le jugement de l'accusé à un groupe de citoyens ordinaires choisis au sein de la population et considérés comme des pairs de l'accusé. De façon plus complexe, le système, au XIX ${ }^{\mathrm{e}}$ siècle, donne lieu à une théorie - la théorie du jury -, avec ses principes et ses règles (conditions pour devenir juré, modes de désignation, systèmes de récusations, modalités de participation au débat judiciaire, modes de délibération et de vote, légitimité des voies de recours, etc.), avec ses promoteurs et ses détracteurs. Dans une thèse publiée en 1849, le Suisse Albert Wessel l'explique en ces termes ${ }^{3}$ :

«La question qui s'agite en Europe depuis le commencement du siècle est posée entre deux théories extrêmes qui sont, pour ainsi dire, chacune toute d'une pièce. Au système des juges seuls, sont liés dans l'opinion publique le système des procédures écrites, les

2 TARDE, Gabriel. Les lois de l'imitation (1890). Paris: Alcan, 1895. p. 343.

3 WESSEL, Albert. Thèse sur le jury criminel. Genève: Jarrys, 1849. p. IV. 
bornes mises à la défense ; à celui du jury se rattachent la publicité, la libre défense, les débats oraux ; le juré n'est plus astreint à des preuves légales, il décide suivant sa conscience. Les deux théories peuvent se soutenir, toutes deux ont aussi leurs côtés faibles; elles ont été violemment attaquées et défendues; et certes la matière d'une discussion pareille est importante, puisqu'il s'agit d'une des premières garanties pratiques de la liberté. »

Les pages qui suivent ont pour objet, à partir de la littérature politique et juridique française des années 1750-1830, de comprendre comment le jury à l'anglaise, qui était unanimement admiré et réclamé dans l'Europe des Lumières, devient, dans la France de la fin de la Révolution, un sujet de controverses et d'ajustements législatifs continuels. Comment l'imitation du modèle anglais a-t-elle été faite ? Comment la transplantation, en dehors de son paysage institutionnel et social d'origine, a-t-elle fonctionné ${ }^{4}$ ? Quels sont les évènements politiques, législatifs ou éditoriaux qui ont alimenté les controverses? Qu'admiret-on? Que rejette-t-on?

$\mathrm{Si}$, dans un premier temps, le jury a conquis les esprits, c'est parce que le modèle politique et criminel anglais était à la mode au XVIII siècle et qu'il a été transposé dans la France révolutionnaire et, par voie de conséquence, dans la plupart des pays d'Europe qui ont été occupés par les troupes françaises. Toutefois, très vite sous la révolution,

4 Sur les concepts d'imitation, de modèle et de transplantation, WATSON, Albert. Legal Transplants: an Approach to Comparative Law. Édimbourg: Scottish Academic Press, 1974; GAUDEMET, Jean. Les transferts de droit. Année sociologique, 1976. p. 29 s.; RODIÈRE, René. Approche d'un phénomène : les migrations de systèmes juridiques. In: Mélanges Marty, Toulouse: PUT, 1978. p. 947 s.; AGOSTINI, Éric. La circulation des modèles juridiques. Revue Internationale de Droit Comparé, 1990. p. 461 s.; MÉNY, Yves (ed.). Les politiques du mimétisme institutionnel. La greffe et le rejet. Paris: L'Harmattan, 1993; LEGRAND, Pierre. The impossibility of "Legal Transplants". Maastricht Journal of European and Comparative Law, 1997. p. 111 s.; SWIECICKA, Paulina. "Connectiones inter iura variorum observamus...". En recherchant l'explication de la notion d'acculturation juridique. In: COPPEIN, Bart; STEVENS, Fred; WAELKENS, Laurent (eds.). Modernisme, Tradition et acculturation juridique. Bruxelles: Iuris Scripta Historica, 2011. p. 7 s.; CAIRNS, John W. Watson, Walton, and the History of Legal Transplants. Georgia Journal of International and Comparative Law, 2014. p. 637 s. 
le jury suscite des doutes parce qu'il ne fonctionne pas comme prévu. Les Thermidoriens et les membres du Directoire cherchent, en vain, à en corriger le fonctionnement. Il devient l'objet d'une première controverse doctrinale lorsque Bonaparte prend le pouvoir et engage la réforme du droit criminel (1799-1808). Maintenu de justesse en 1808, le jury criminel fait l'objet d'une seconde controverse doctrinale après la chute de Napoléon et la restauration de la monarchie (1815-1830). Les nombreux plaidoyers en faveur du jury à l'anglaise sont relativisés par certaines études menées sur place. L'analyse de cette littérature juridique montre qu'en matière criminelle, le système pénal anglais est un système controversé à cause des qualités et des défauts techniques de l'institution, mais à cause aussi des évènements politiques et de l'état de la criminalité.

\section{Les Lumières et L'ENGOUEMENT POUR LE JURY À L'ANGLAISE}

En 1750, la procédure criminelle française se distingue nettement de la procédure anglaise. Fidèle à la tradition du ius commune, l'ordonnance française de 1670 organise la procédure autour de l'instruction. Résumons. Lorsqu'un crime a été commis, il déclenche l'action publique confiée au parquet. Le procureur du roi ou du seigneur engage les poursuites sur la base d'une plainte, d'une dénonciation ou d'une rumeur. Il requiert du magistrat instructeur qu'il constate le crime, qu'il auditionne les témoins, qu'il fasse réaliser des expertises, qu'il interroge les suspects. Au terme de cette instruction préparatoire, le parquet décide de la suite. Il peut interrompre les poursuites. Il peut aussi régler à l'ordinaire lorsqu'il s'agit d'une infraction légère (injure, blessure minime, vol modique) et, dans ce cas, le procès se poursuit selon la procédure civile, afin d'indemniser la victime. Il peut encore régler à l'extraordinaire, pour les infractions qui méritent une peine afflictive (qui fait souffrir le condamné) ou infâmante (qui porte atteinte à l'honneur du condamné). Dans ce cas, le magistrat instructeur procède, vis-à-vis de l'accusé, et sans présence d'un avocat, à une instruction définitive qui complète et confirme l'instruction préparatoire. Puis, l'accusé passe en jugement devant les juges, sans avocat et sans jury. Si la preuve du crime est rapportée (l'aveu ou deux témoins minimum), le juge doit condamner l'accusé. Sinon, il doit renvoyer l'accusé hors de cours : la conscience du juge s'efface devant le système des preuves 
légales. En revanche, sauf si la loi prévoit une peine précise, le juge jouit de l'arbitrium. Il peut arbitrer la peine en fonction de la personnalité du coupable et des circonstances du crime : la légalité stricte des délits et des peines s'efface devant la conscience du juge. Sauf exception, les jugements criminels sont rendus à charge d'appel devant les juridictions supérieures. Quant au roi, source de toute justice, il peut toujours faire casser un arrêt d'appel ou gracier un condamné. Cette procédure, inquisitoire, secrète, non contradictoire, repose sur les éléments de l'instruction qui, en principe, doit révéler la vérité et ne conduire devant les juges que ceux pour qui la présomption de culpabilité est très forte ${ }^{5}$. Dans ce système, le suspect et l'accusé ne sont pas dépourvus de moyens de défense, mais ces moyens sont fondés sur les devoirs des magistrats et le respect de la procédure et plus que sur les droits de la défense ${ }^{6}$.

En Angleterre, au XVIII ${ }^{e}$ siècle, l'action judiciaire ne peut être engagée et conduite par un officier public que par exception. C'est le cas pour les crimes les plus graves (trahison, sédition, fausse monnaie) et pour de nombreuses atteintes aux biens. Dans ce cas, la victime s'efface devant le magistrat qui conduit l'affaire jusqu'à son terme ${ }^{7}$. Mais, en principe, l'action repose sur la victime qui peut l'entamer de trois façons ${ }^{8}$ : l'appeal, l'information et surtout l'indictment, le procédé le plus célèbre. Cet indictment est le résultat d'une enquête préliminaire après une plainte auprès du juge de paix. Le suspect est présenté devant le grand jury, composé de douze à vingt-trois juges de paix du comté. Ils examinent les charges qui pèsent contre le suspect. Si douze grands jurés au moins

5 CRÉPIN, Marie-Yvonne. Ordonnance criminelle dite de Saint-Germain-enLaye, août 1670. In: HAUTEBERT, Joël; SOLEIL, Sylvain (eds.). La procédure et la construction de l'État en Europe (XVI $I^{\mathrm{e}}$ XIX ${ }^{\mathrm{e}}$ siècles). Recueil de textes, présentés et commentés. Rennes: PUR, 2011. p. $476 \mathrm{~s}$.

6 ASTAING, Antoine. Droits et garanties de l'accusé dans le procès criminel d'Ancien Régime ( $X V I^{e}-X V I I I^{e}$ siècle). Audace et pusillanimité de la doctrine pénale française. Aix-en-Provence: PUAM, 1999; BLOT-MACCAGNAN, Stéphanie. Procédure criminelle et défense de l'accusé à la fin de l'Ancien Régime. Rennes: PUR, 2010.

7 SMITH, Bruce P. The myth of private prosecution in England, 1750-1850. In: DUBBER Markus D.; FARMER Lindsay (eds.). Modern Histories of Crime and Punishment. Stanford: SUP, 2007. p. 151 s.

8 BAKER, John H. An Introduction to English Legal History. Londres: Butterworths, 2000. p. $70 \mathrm{~s}$. 
estiment que le dossier justifie la conduite d'un procès, ils rédigent un acte d'accusation (count of indictment) et l'affaire est jugée lors des assises. Durant le procès, le petit jury, composé de douze citoyens, écoute les débats contradictoires entre l'accusation et la défense. Le juge résume les débats, parfois en donnant sa propre opinion, et les douze jurés doivent décider, à l'unanimité, de la culpabilité de l'accusé9. Lors du procès, prévaut une procédure accusatoire, publique et contradictoire, qui repose, en principe, sur un combat à armes égales entre l'accusation et la défense. En pratique, comme l'explique Louis de Carbonnières à partir des observateurs de l'époque, ce système était loin d'être parfait ${ }^{10} \ldots$

Toutefois, à compter de 1750, sur le continent européen, les philosophes et les magistrats éclairés se passionnent pour l'institution du jury. Ils en parlent, ils en discutent, ils en font un modèle ${ }^{11}$. En France, les auteurs parmi les plus en vogue, Montesquieu, Voltaire, Jaucourt, Condorcet, Dupaty, Marat, Linguet, Servan, Letrosne ou Delolme publient des ouvrages et des pamphlets exaltés ${ }^{12}$. Lorsqu'on consulte les dix volumes de la Bibliothèque philosophique dirigée et publiée par Brissot de Warville, de 1782 à 1785, on mesure la force et la nature de cet enthousiasme ${ }^{13}$. Dans le préliminaire du premier tome, Brissot salue les grands réformateurs européens et les constitutions des nouveaux États américains, notamment la Pennsylvanie, qui prévoient le double jury, le premier chargé de l'instruction « prompte et publique, par des jurés impartiaux du pays », le second chargé de garantir qu'aucun homme «ne peut être privé justement de sa liberté, qu'en vertu des lois du pays

9 BEATTIE, John. Crime and the Courts in England, 1660-1800. Oxford: Clarendon Press, 1986; KING, Peter. Crime, Justice and Discretion in England, 17401820. Oxford: OUP, 2000.

10 CARBONNIÈRES, Louis (de). Les destinées du jury français et du jury anglais (XVIII ${ }^{\mathrm{e}}$-XIX ${ }^{\mathrm{e}}$ siècles). Les Cahiers de la Justice. Paris, 2012. p. 83 s.

11 BLOT-MACCAGNAN, Stéphanie ; SOLEIL, Sylvain. Aufklärung und Justizreform. In: MAYENBURG, David (von); COLLIN, Peter; DECOCK, Wim; GROTKAMP, Nadine; SEENLENTAG, Anna (eds.). Geschichte der Konfliktregulierung in Europa - Ein Handbuch. Berlin: Springer, 2021. p. 223 s.

12 CARBASSE, Jean-Marie. Histoire du droit pénal et de la justice criminelle. Paris: PUF, 2000. p. 355.

13 TIYAL, Samyr. Jacques-Pierre Brissot de Warville, genèse d'une pensée (17701789). Lille: ANRT, 1997. 
et du jugement de ses pairs $»^{14}$. Au tome 3 , avant de décrire les origines et le fonctionnement du jury criminel en Pennsylvanie, il s'exclame ${ }^{15}$ : «Que de précautions prises, pour défendre cette liberté politique, dont on ne connaît plus que le nom en Europe ! ». Au tome 5, Brissot publie le célèbre Prix de la justice et de l'humanité, dans lequel Voltaire s'écrie ${ }^{16}$ : « En Angleterre, île fameuse par tant d'atrocités et par tant de bonnes lois, les jurés étaient eux-mêmes les avocats de l'accusé. [...] En France, le code criminel paraît dirigé pour la perte des citoyens ; en Angleterre, pour leur sauvegarde ». Au tome 7, Brissot publie les Réflexions sur quelques points de nos lois, dans lesquelles l'ancien magistrat Servan critique la procédure criminelle française et s'adresse à la nation suisse ${ }^{17}$ :

«C'est de vous que le continent de l'Europe attend le grand exemple de la procédure par les jurés, et du jugement par les pairs. Cette loi est reléguée sur une île [allusion à l'Angleterre], dont la guerre et le préjugé nous séparent encore plus que la mer. Mais dans votre patrie, mêlée parmi vous, elle n'aura plus à nos yeux ce visage ennemi. Dès longtemps vous êtes nos frères, et peut-être vous nous communiqueriez vos bonnes lois criminelles, comme nous vous avons communiqué nos privilèges. »

En bref, les philosophes et les magistrats éclairés admirent tous le jury qu'ils opposent au modèle inquisitoire. Le système français est condamné soit à l'occasion de procès partiaux et mal conduits, soit à l'occasion de châtiments cruels ou disproportionnés. Le système anglais est admiré parce qu'il serait rapide, libéral, impartial, ne produirait ni excès, ni erreurs judiciaires. En fait, ces auteurs adoptent une posture politique. Ils ont retenu de L'esprit des lois de Montesquieu (1748), que le jury criminel constitue un fondement des libertés publiques et individuelles ${ }^{18}$. $\mathrm{Si}$, affirment-ils, la liberté consiste à protéger la vie, l'honneur et les biens

14 BRISSOT. Bibliothèque philosophique..., v. 1. Paris: Desauges, 1782-1785, p. XXXIII-XXXIV.

15

Ibid., v. 3. p. 246-248.

16 Ibid., v. 5. p. 91.

17 Ibid., v. 7. p. 277.

18 TILLET, Édouard. La constitution anglaise, un modèle politique et institutionnel dans la France des Lumières. Aix-Marseille: PUAM, 2001. p. 449 s. 
des citoyens et les mettre à l'abri des lois tyranniques, il faut toujours craindre les juges trop puissants ; il ne faut jamais redouter les jurés parce que ce sont des citoyens comme les autres. Par voie de conséquence, les philosophes et les magistrats éclairés font du jury à l'anglaise un modèle à imiter, mais un modèle idéalisé, fantasmé, purgé des défauts réels que l'on connaissait peu ou mal à l'époque.

\section{Les Révolutionnaires et L'ADOption du JURy À l'ANGLAISE}

On comprend pourquoi, au moment où commence la Révolution française (printemps 1789), l'Assemblée nationale constituante met la réforme de la justice criminelle à l'ordre du jour en s'inspirant, pour l'essentiel, des philosophes des Lumières et du modèle anglais ${ }^{19}$. La loi d'organisation judiciaire des 16-24 août 1790 dispose : « La procédure par jurés aura lieu en matière criminelle » (titre II, art. 15). La réforme, adoptée dans l'enthousiasme général, s'inscrit dans un nouvel équilibre constitutionnel $^{20}$. La justice n'est plus rendue au nom du roi mais au nom du peuple français. Le roi n'a plus le pouvoir d'intervenir dans le cours de la justice ou de gracier un condamné. Les juges, désormais élus, sont confinés à un rôle secondaire ; ils ne doivent qu'appliquer la loi aux faits, ils doivent juger comme des « automates » (Beccaria), ils doivent n'être que « la bouche de la loi » (Montesquieu). Le jury, quant à lui, représente la nation dans la fonction judiciaire, comme l'Assemblée représente la nation dans la fonction législative ${ }^{21}$.

19 DRUGEON, Laurent. Les sources du droit procédural révolutionnaire : une voie française ? In: HAUTEBERT, Joël; SOLEIL, Sylvain (eds.). Modèles français, enjeux politiques et élaboration des grands textes de procédure en Europe, v. 2. Paris: EJT. p. $13 \mathrm{~s}$.

20 SOLEIL, Sylvain. Lex Imperat : Creation and Exportation of the French Model of the Legality Principle (18th-19th C.). In: MARTYN, Georges; DUBBER Marcus D.; PIHLAJAMÄKI Heikki (eds.). From the Judge's Arbitrium to the Legality Principle - Legislation as a Source of Law in Criminal Trials. Berlin: Duncker \& Humblot, 2013. p. 145 s.

${ }^{21}$ RAY, Jean. La Révolution française et la pensée juridique : l'idée de règne de la loi. Revue Philosophique, 1939. p. 363 s.; HALPÉRIN, Jean-Louis. Haro sur les hommes de loi, Droits, 1993. p. 55 s.; PADOA-SCHIOPPA, Antonio (ed.). 
Le code des 29 septembre - 21 octobre 1791 établit la procédure criminelle sur de nouvelles bases. Le système devient accusatoire. L'unité du parquet, organisé autour du procureur du roi, est brisée au profit d'un accusateur public (un citoyen élu), chargé de porter l'accusation contre l'accusé, et d'un commissaire du roi (un magistrat nommé par le roi et inamovible) chargé de veiller à l'exacte application de la loi et de la procédure dans chaque juridiction. La phase d'instruction est confiée, au niveau du canton, au juge de paix assisté de notables. La phase d'accusation, au niveau du district, est confiée au jury d'accusation, inspiré du grand jury anglais, qui décide si les charges à l'égard du suspect suffisent pour le juger. La phase de jugement, au niveau du département, est confiée au jury criminel, inspiré du petit jury anglais, qui décide si l'accusé est coupable ou non. L'appel est aboli puisque le jury juge au nom de la nation tout entière. Le principe des preuves légales disparaît au profit de la seule conviction du jury. L'arbitrium des juges est remplacé par le principe de la légalité stricte des délits et des peines.

Lors des débats à l'Assemblée, les questions les plus agitées concernent la nature et les limites des pouvoirs des jurés, les conditions pour être jurés et le nombre de voix nécessaire pour prononcer une condamnation. Malgré les mises en garde de ceux qui connaissent les défauts du jury à l'anglaise ${ }^{22}$, le système est voté par les députés, accepté par le roi, mis en vigueur au début de l'année $1792^{23}$. Et, malgré plusieurs aménagements, il va durer jusqu'en décembre 1810 .

The Trial Jury in England, France, Germany, 1700-1900. Berlin: Duncker \& Humblot, 1987. p. 75 s.

CARBONNIÈRES, Louis (de). Le comte de Lauraguais, anglomane isolé, face au jury révolutionnaire. Revue Historique de Droit Français et Etranger, 2013. p. 283 s.; sur la différence entre les deux systèmes : BERGER, Emmanuel. The Criminal Jury in England and France in the late $18^{\text {th }}$ Century. Historiographical Issues and Research Perspectives of Popular Justice. In: DELIVRÉ, Emilie; BERGER, Emmanuel (eds.). Popular Justice in Europe $\left(18^{\text {th }}-19^{\text {th }}\right.$ Centuries). Berlin: Duncker \& Humblot, 2014, p. 71 s.

LAFON, Jacqueline-Lucienne. La Révolution française face au système judiciaire d'Ancien Régime. Genève, Droz, 2001; MARTUCCI, Roberto. La Costituante ed il problema penale in Francia (1789-1791), 1. Alle Origine dei processo accusatorio : I decreti Beaumetz. Milan: Giuffrè, 1984; BERGER, Emmanuel. La justice pénale sous la Révolution. Les enjeux d'un modèle judiciaire libéral. Rennes: PUR, 2008. 
Techniquement, comment fonctionnait le double jury? Après la phase d'instruction, si la victime ou l'accusateur public décidaient d'accuser un suspect, le directeur du jury, un magistrat élu, rédigeait un acte d'accusation qu'il soumettait au jury d'accusation, composé de huit citoyens. Au cas où le directeur du jury refusait de présenter l'affaire aux jurés, la victime pouvait le faire en rédigeant elle-même l'acte d'accusation. Ce jury d'accusation était réuni au tribunal du district et pouvait décider soit qu'il n'y avait pas lieu de poursuivre le suspect était remis en liberté - soit qu'il y avait lieu d'accuser le suspect et l'affaire était transmise, avec l'accusé et l'acte d'accusation, au tribunal criminel. Là, devant les juges élus et douze jurés tirés au sort, se déroulaient les débats publics et contradictoires. On écoutait la lecture de l'acte d'accusation, les arguments de l'accusateur public et la défense de l'accusé qui pouvait être représenté par le défenseur de son choix. Puis, les douze jurés se retiraient dans une salle à part, hors la présence des juges, pour délibérer. Chacun d'eux se rendait seul, à tour de rôle, dans la chambre du conseil où étaient disposées des boîtes. Il y avait autant de boîtes que de questions posées au jury. Le premier juré, en présence d'un juge, répondait à la première question (les faits constituent-ils un crime ?) en glissant une boule blanche (en faveur de l'accusé) ou une boule noire (contre l'accusé) dans la première boîte. S'il avait déposé une boule blanche, c'est qu'il estimait qu'il n'y avait pas eu de crime et il quittait donc la chambre du conseil, puisqu'il n'avait pas à répondre aux questions suivantes. S'il déposait une boule noire, on lui demandait de répondre, par une boule blanche ou une boule noire, à la deuxième question (l'accusé est-il l'auteur de ce crime ?), et ainsi de suite pour chacune des questions posées. On procédait de cette façon avec chacun des douze jurés afin d'éviter que les jurés soient soumis à un vote collectif. Prenons l'exemple d'un vol de ruches d'abeilles commis en $1798^{24}$. Les coupables ayant avoué leur crime, le directeur du jury d'accusation dresse l'acte d'accusation le 17 octobre, le jury d'accusation vote en faveur de l'accusation le 21 octobre, et les accusés sont traduits devant le tribunal criminel le 6 décembre 1798. Les jurés,

24 Archives d'Ille-et-Vilaine, L2823 \& L3072, Rouxel, accusateur public, c/ Rieux, père et fils, 1798. 
à tour de rôle, doivent répondre, à l'aide de leurs boules blanches ou noires, aux questions suivantes :

Est-il constant qu'une ruche d'abeilles fût volée à Michel Batois?

Luc Rieux est-il un des auteurs dudit vol?

L'a t-il commis méchamment et dans l'intention du vol ? [...]

Le vol a-t-il été commis la nuit?

Durant la procédure de vote, la règle était la suivante : dès que le juge constatait la présence de trois boules blanches dans les boîtes, il interrompait la procédure car trois boules blanches signifiaient trois votes en faveur de l'accusé, ce qui suffisait pour l'acquitter. Si, au contraire, il n'y avait pas plus de deux boules blanches dans l'ensemble des boîtes, l'accusé était déclaré coupable. En janvier 1792, lorsque le système entre en vigueur, chacun espère qu'il va bien fonctionner et qu'il va permettre d'assurer l'équilibre entre le respect des droits de l'accusé et le respect de l'ordre public, entre la défense des libertés individuelles et la défense de l'ordre social.

\section{LA PRATIQUE JUDICIAIRE ET LE PROBLÈME DES ACQUITTEMENTS SCANDALEUX}

Très vite, en réalité, l'enthousiasme pour le jury va laisser place aux doutes. Pour le comprendre, il faut revenir sur les évènements de la période 1792-1794. Le durcissement des tensions politiques (entrée en guerre de la France contre l'Autriche et la Prusse, déchéance du roi, avènement de la République, exécution du roi, etc.) et la dynamique révolutionnaire elle-même conduisent la nouvelle assemblée (la Convention, élue en septembre 1792) et ses comités de salut public et de sûreté générale à tout interpréter en termes idéologiques : pour ou contre la Révolution, pour ou contre la République. Tout ce qui ne se plie pas au nouvel ordre politique est désormais interprété comme une attitude contrerévolutionnaire. Sur le plan judiciaire, le pouvoir exige des tribunaux une application des lois stricte, docile et conforme à l'idéologie. Vis-à-vis des crimes à caractère politique (conspiration, rébellion, liens de parenté ou 
d'amitié avec les nobles ou les prêtres, etc.), le problème est résolu avec la création des tribunaux criminels extraordinaires et des commissions militaires qui jugent sans jury. Ce sont ces institutions qui, du printemps 1793 à l'automne 1794, vont faire régner la terreur en France ${ }^{25}$. Pour les crimes de droit commun, le problème restera insoluble ${ }^{26}$. D'un côté, le pouvoir politique veut que sa politique criminelle soit appliquée par les tribunaux criminels. De l'autre, les jurys d'accusation refusent d'accuser les suspects et les jurys criminels refusent de condamner les accusés dans une proportion inquiétante. Comme l'appel de leurs décisions est impossible, le pouvoir politique demeure impuissant. La seule parade est l'annulation des décisions. De fait, de mars 1793 à octobre 1795, la convention va annuler deux cents décisions des tribunaux criminels.

À l'époque, au ministère de la justice et dans les comités révolutionnaires, ce problème est connu sous le nom des acquittements scandaleux. Essayons de comprendre, à partir des travaux de Robert Allen, Lynn Hunt, Gilles Landron et, plus récemment, Xavier Rousseau et Emmanuel Berger. Dans le nouvel équilibre pénal, le juge n'est pas libre de personnaliser les peines selon ce que lui dicte sa conscience. On craint son arbitraire. C'est donc la loi, le Code pénal de 1791, qui fixe la liste stricte et précise des peines qui devront être prononcées par les juges si le jury a déclaré l'accusé coupable. Par exemple, l'article 6 du Titre 2, Section 2 du Code prévoit que la peine pour un vol sera de huit années de fers, que le coupable soit une indigente ou un criminel aguerri, que le vol concerne un pain ou toutes les économies de la victime. L'article 7 fixe les cinq circonstances aggravantes pour lesquelles cette peine sera augmentée de deux années supplémentaires. De sorte qu'un vol de pain (8 ans), commis de nuit $(+2)$, dans une maison habitée $(+2)$, doit

25 BROWN, Howard G. Ending the French Revolution: Violence, Justice, and Repression from the Terror to Napoleon. Charlottesville: UVP, 2006; MARI, Éric (de). La mise hors de la loi sous la Révolution française (1793-an III). Paris: LGDJ, 2015; BOULANT, Antoine. Le tribunal révolutionnaire. Punir les ennemis du peuple. Paris: Perrin, 2018.

26 CARBASSE, Jean-Marie. État autoritaire et justice répressive : l'évolution de la législation pénale de 1789 au Code de 1810. In: All'ombra dell'aquila imperiale. Rome: Archivi di Stato, 1994. p. 325 s.; PASSANO, Mario (da). Emendare $o$ intimidire ? La codificazione del diritto penale in Francia e in Italia durante la Rivoluzione e l'Impero. Turin: Giappichelli, 2000. p. 101 s. 
être puni de 12 années de fers, c'est-à-dire une peine manifestement disproportionnée.

Les jurés ont souvent été confrontés à ce genre de situations où, d'un côté, l'accusé était évidemment coupable, où, d'un autre côté, la peine leur paraissait démesurée. Dans certains cas, ils ont donc préféré ne pas poursuivre ou acquitter plutôt que d'assumer une peine injuste ${ }^{27}$. En outre, certains jurés pouvaient avoir tendance à excuser des crimes qu'ils avaient eux-mêmes commis. De plus, tous n'étaient pas forcément capables de comprendre les questions complexes qui leur étaient posées ou de dissocier la culpabilité de l'innocence. Pour un juré, déposer une boule blanche ne signifiait pas forcément qu'il était convaincu de l'innocence de l'accusé, mais plutôt qu'il n'était pas convaincu de sa culpabilité, et que le doute devait lui profiter. Par ailleurs, beaucoup n'étaient en phase ni avec les crimes et les peines prévus dans le code de 1791, ni avec les nouvelles incriminations à caractère politique ${ }^{28}$. Et puisqu'on demandait à chaque juré de suivre "votre conscience et votre intime conviction, avec l'impartialité et la fermeté qui conviennent à un homme libre », de nombreux jurés ont agi... comme des hommes libres. Et puisqu'il suffisait de trois boules blanches pour acquitter un accusé, le système a finalement tourné en faveur des accusés.

Les études menées dans les archives judiciaires le montrent. Emmanuel Berger, qui a examiné les territoires belges réunis à la France, aboutit à un taux de 33\% d'affaires où les jurys d'accusation ont refusé de mettre le suspect en accusation, soit 300 affaires environ sur $900^{29}$. Le taux monte à $54 \%$ pour les affaires à connotation politique. L'étude des verdicts des jurys criminels, menée dans des départements divers, aboutit aux mêmes résultats. Robert Allen, qui a étudié les verdicts rendus dans seize départements entre 1792 et 1811, aboutit à un taux moyen de $45 \%$

27 LANDRON, Gilles. À propos de la "commisération abusive" du jury criminel de la Révolution. Histoire de la Justice, 1994. p. 73 s.; SCHNAPPER, Bernard. L'activité du tribunal criminel de la Vienne, 1792-1800. In: La Révolution et l'ordre juridique privé : rationalité ou scandale?, v. 2. Paris: PUF, 1988. p. 623 s.

28 ALLEN, Robert. Les tribunaux criminels sous la révolution et l'Empire (17921811). Rennes: PUR, 2005. Chap. II, § 17 s.

29 BERGER, Emmanuel. Ordre public et poursuites criminelles sous le Directoire (1795-1799). Annales Historiques de la Révolution Française, 2007. p. 146. 
d'acquittements, soit environ 8500 acquittements pour 19000 affaires jugées $^{30}$. Ce taux, selon Jean-Louis Debauve, monte même à $65 \%$ dans le département du Morbihan ${ }^{31}$. Robert Allen et Xavier Rousseaux, qui ont comparé les chiffres, expliquent que ces taux restent comparables durant toute la période (1793-1799), qu'il n'y a aucune corrélation entre la sensibilité politique des jurys et le taux d'acquittements, et que ce taux est plus élevé dans les affaires à caractère politique que dans les affaires classiques de vol ou de violence ${ }^{32}$.

De façon rétrospective, on peut estimer que les jurés ont parfaitement joué leur rôle : défendre les libertés publiques en protégeant les citoyens contre les lois liberticides. Mais, de 1793 à 1799, le pouvoir politique ne voit pas du tout les choses de la même façon. À leurs yeux, les jurys d'accusation et de jugement sont suspectés d'être rebelles à la loi ${ }^{33}$. À compter de la chute de Robespierre (juillet 1794), la Convention, puis le nouveau régime qu'elle met en place, le Directoire (1795-1799), vont chercher à rectifier la situation ${ }^{34}$. Les discours contre les jurés qui, par leur indulgence coupable, conspireraient contre la République se multiplient à tous les niveaux. Les listes de jurés sont purgées des éléments douteux. Les lois multiplient le nombre d'affaires jugées par des tribunaux sans jury. L'acquittement avec trois boules blanches est modifié au profit de l'unanimité (pour ou contre la culpabilité de l'accusé) ou, si au bout de vingt-quatre heures assemblé le jury n'a pas réussi à se mettre d'accord, à la majorité. Mais rien n'y fait. Le taux d'acquittements à la veille du coup d'État de Napoléon Bonaparte reste toujours aussi élevé.

30 ALLEN. Les tribunaux criminels (op. cit.), Chap. II, §5.

31 DEBAUVE, Jean-Louis. La justice révolutionnaire dans le Morbihan (17901795). Paris: Debauve, 1965.

32 ALLEN. Les tribunaux criminels (op. cit.), Chap. II, §67 s.; ROUSSEAUX, Xavier. Politique judiciaire, criminalisation et répression. La révolution des juridictions criminelles (1792-1800). In: MARTIN, Jean-Claude (ed.). La Révolution à l'œuvre. Perspectives actuelles dans l'histoire de la Révolution française. Rennes: PUR, 2005. §38 s.

33 SCHNAPPER, Bernard. Le jury criminel. In: BOUCHER, Paul (ed.). Une Autre Justice, 1789-1799. Paris: Fayard, 1989. p. 170.

34 CRÉPIN. Marie-Yvonne. Les Thermidoriens et la reconstruction juridique. In: La Vendée. Après la Terreur, la reconstruction. Paris: Perrin. 1997. p. 191 s. 


\section{Le Consulat et la première controverse doctrinale sur Le JuRY}

Napoléon Bonaparte prend le pouvoir en novembre 1799 (brumaire an VIII) et supprime le système du Directoire au profit du Consulat. Il met au point une constitution qui donne le rôle principal au pouvoir exécutif et qui lui permet de relancer les chantiers de la codification. En matière criminelle, Bonaparte est décidé à examiner ce qui n'a pas fonctionné et à faire les réformes nécessaires. L'une des questions majeures est celle du maintien du jury à l'anglaise. C'est pourquoi, de 1799 à 1804, on assiste à une floraison d'ouvrages et d'articles, pour ou contre l'institution du jury criminel. En l'an VIII, Granger, substitut du commissaire du gouvernement près le tribunal criminel de la Seine, qui s'est rallié à Bonaparte au nom des « amis de l'humanité et des ennemis du désordre et de l'anarchie », se propose de faire la liste des problèmes qu'il a observés dans l'exercice de ses fonctions. À propos des jurés, il annonce que « le moment de dire de grandes vérités est arrivé ${ }^{35}$. La publicité des listes de jurés a, affirmet-il, offert aux accusés l'occasion d'envoyer aux jurés des mémoires manuscrits ou imprimés pour les influencer. Les listes ont été faites sans rigueur et contiennent parfois les noms d'hommes qui sont décédés ou qui ont quitté le département. Le tirage au sort désigne des hommes qui ne savent pas lire les documents qu'on leur présente, ou qui, étant sourds, ne peuvent pas siéger utilement, ou qui, stupides ou entêtés, s'avèrent incapables de tenir leur rôle. Certains annoncent hautement « qu'ils n'entendent rien à toutes les questions qu'on leur propose ; disent que ce n'est pas là leur métier ; qu'il faut leur laisser faire celui qu'ils ont appris. Ils ne donnent leurs boules alors, que parce qu'ils y sont forcés, et les donnent avec humeur et au hasard ${ }^{36}$. Le nombre de questions posées au jury, ajoute Granger, complexifie les choses, ralentit les délibérations et suscite des risques de cassation. L'ancien système des trois boules blanches était catastrophique ${ }^{37}$ :

35 GRANGER. Idée des abus existants dans l'ordre de procéder en matière criminelle. Paris: Guerbart, 1800. p. 80-81.

36 Ibid. p. 116.

37 Ibid. p. 136-137. 
«Il devait résulter, et il est résulté, de l'inégalité de cette lutte entre les accusés et la société, que celle-ci a persévéramment souffert, tandis que les autres ont impunément joui du fruit de leurs rapines et du plus affreux brigandage. Il est résulté, de cette inégalité, que des hommes évidemment coupables, et à la conviction desquels il ne manquait absolument que leur aveu, ont été scandaleusement acquittés, et se sont publiquement amusés de l'erreur ou de l'ineptie de ceux de leurs jurés qui venaient de concourir à leur absolution. »

Quant au nouveau système du vote à l'unanimité, il est contraire à la morale car on pousse les jurés à céder par lassitude ou impatience. Granger ne propose pas à Bonaparte la suppression du jury, mais des réformes de fond.

Quelques mois plus tard, le 5 janvier 1801, l'Institut de France, dans sa formation des sciences morales et politiques, organise un concours : «Quels sont les moyens de perfectionner l'institution du jury? ». Cette technique du concours était assez courante dans l'Europe des Lumières car elle permettait de mobiliser les intelligences et de recueillir des idées sur des sujets à l'ordre du jour. D'ailleurs, ce sont de parfaits inconnus qui vont s'illustrer lors de ce débat et dont les arguments respectifs vont, avec les opinions des tribunaux, occuper l'espace public. En avril 1802, l'Institut décerne deux prix, l'un au citoyen Canard, ancien professeur de mathématiques à l'école centrale de Moulins, l'autre au citoyen Bourguignon, juge au tribunal criminel de la Seine. Ces mémoires, et plusieurs autres, sont alors imprimés ${ }^{38}$. L'un et l'autre, favorables au jury, se désolent à propos des difficultés rencontrées par l'institution et proposent des solutions. Dans un deuxième mémoire, lu à l'académie de législation en décembre 1803, Bourguignon s'en prend d'ailleurs avec virulence aux détracteurs du jury qui ont prospéré depuis 1802 et la publication de son premier mémoire ${ }^{39}$ :

38 CANARD. Moyens de perfectionner le jury. Moulins: Vidalin, 1802; BOURGUIGNON. Mémoire sur les moyens de perfectionner en France l'institution du jury. Paris: Maradan, 1802; BONNET. Du jury en France. Paris: Maradan, 1802; LOUVET. Discours... sur cette question posée en l'an IX : quels sont les moyens de perfectionner l'institution du jury? Paris: Chaumerot, 1808.

39 BOURGUIGNON. Deuxième mémoire sur l'institution du jury. Paris: Patris, 1804. p. 2. 
« Depuis lors, j'ai entendu des jurisconsultes et des magistrats du plus grand mérite, révoquer en doute la supériorité de cette procédure, ne la considérer que comme une brillante théorie, qui, mise en pratique, procure l'impunité à un grand nombre de criminels, et porte par conséquent le désordre le plus funeste dans la société. Le peu de succès, disent-ils, qu'elle a obtenu en France, depuis qu'elle y est observée, prouve, jusqu'à l'évidence, que, fûtelle bonne, elle ne peut convenir à nos mœurs. »

Ce que redoute Bourguignon, c'est que ces juristes et ces magistrats d'Ancien Régime ne réussissent à convaincre l'opinion publique et le pouvoir politique d'abolir le jury pour récupérer le pouvoir de juger, seuls, les accusés. Treize mois plus tôt, Scipion Bexon, un magistrat, avait lui aussi souligné cet enjeu. S’il est vrai, admet-il, que le problème est en partie lié au caractère des jurés, il faut également convenir qu'il découle aussi de l'attitude des magistrats qui ont pratiqué l'ancien système. Ils se montrent souvent défavorables à l'institution car leur « habitude contractée de juger dans le secret de l'ancienne procédure, avec ses formes acerbes et sa dureté, sympathise peu avec la candeur et la simplicité des formes de l'institution du jury $\gg^{40}$.

Dans l'autre sens, Gach, président du tribunal de première instance de Figeac, dénonce le jury à partir des faits qui, à l'opposé des doctrines du siècle des Lumières, ont montré l'échec du jury. Il se propose donc de s'appuyer sur « ce qui est, ce que j'ai vu, ce que chacun a vu », et d'établir ${ }^{41}$ :

«Que l'institution du jury n'est qu'un beau rêve de la philosophie, impossible à réaliser parmi nous ; que le sol français, d'ailleurs si fécond en hommes célèbres ou estimables dans tous les genres de talent et de mérite, ne produira jamais de bons jurés, et que l'obstacle est dans le caractère, les mœurs, les vices, et jusques dans les vertus de la nation. »

Le pamphlet de Gach a ceci de percutant qu'il conteste non le jury en soi, mais la transplantation faite en France. Le jury d'origine

40 BREXON. Développement de la théorie des lois criminelles. Paris: Garnery, 1802. p. 45.

41 GACH. Des vices de l'institution du jury en France. Paris: Petit, 1804. p. v. 
anglaise, affirme-t-il, ne correspond pas au caractère du peuple français. En effet, l'institution ne peut fonctionner que si les citoyens sont honorés d'y participer et s'ils ont l'instruction et les compétences requises. Or, combien de fois a-t-on vu des jurés s'absenter la veille du procès, se déclarer malades ou se cacher dans leur maison pour éviter de siéger. Courageux et magnanime sur le champ de bataille, le Français, explique Gach, n'a pas les mêmes dispositions quand il s'agit d'autres affaires publiques. Il privilégie toujours ses intérêts particuliers et ses plaisirs au détriment de l'intérêt général. Il est léger, frivole, irréfléchi, prompt à décider. Il a tendance à excéder les bornes du pouvoir d'un juré. Il a du mal à respecter la loi. Il a un penchant à l'indulgence. En outre, le peuple français, le peuple le plus poli, le plus spirituel et le plus aimable de la terre, est aussi le moins instruit. S'élever à propos des questions politiques ne l'intéresse pas. Bref, il ne possède pas les qualités requises pour faire un bon juré. Bref, « le vice dominant et fondamental de l'institution était dans son opposition avec les mœurs françaises et le caractère national $\gg^{42}$. En termes de théorie du droit, la transplantation de l'institution anglaise est un échec. Gach conclut sur «l'urgente nécessité de la supprimer ».

En 1804, lorsque La Revue Philosophique, Littéraire et Politique publie une réplique à Gach ou lorsque Porcher défend le jury, c'est avec angoisse car, affirment l'une et l'autre, désormais, l'institution est contestée par les autorités publiques elles-mêmes ${ }^{43}$.

\section{L'EMPIRE, LA SUPPRESSION DU JURY D'ACCUSATION ET LE MAINTIEN DU JURY CRIMINEL}

De fait, les projets de réforme criminelle ont évolué dans un sens défavorable au système français et au modèle du jury à l'anglaise. Déjà en janvier 1801, Bonaparte a fait changer les règles concernant l'acte d'accusation présenté au jury d'accusation qui sera désormais rédigé par le commissaire du gouvernement (nommé par le pouvoir exécutif)

42 Ibid., p. 24, 43 et 90.

43 ANONYME. Aux rédacteurs de la Revue. La Revue Philosophique, Littéraire et Politique, 1804-1805. p. 12 s.; PORCHER. L'excellence de l'institution du jury. Orléans: Guyot, 1804. p. II. 
et non plus par le directeur du jury (un juge élu). Une commission est instituée en mars 1801 pour rédiger un projet de code comprenant à la fois le droit pénal et la procédure. Elle est animée par cinq hommes qui cherchent à trouver l'équilibre entre la conservation du jury et une logique d'efficacité, de répression, de reprise en main de la société française. Ils participent de ce basculement décrit par Stefano Solimano : « une grande partie des juristes napoléoniens, de 1789 à Brumaire, avaient en commun le même parcours spirituel, la même évolution idéologique : de l'illusion au désenchantement $»^{44}$. Ces hommes, désabusés, réactionnaires, vieillis, expriment désormais une grande défiance à l'égard des hommes en société. En matière criminelle, ils réclament donc de la rigueur, de la sévérité, des châtiments spectaculaires. Toutefois, ils veulent conserver certains acquis $^{45}$. Oudard, l'un des deux rapporteurs, admire d'ailleurs toujours le modèle anglais ${ }^{46}$. Leur projet maintient donc le double jury sous réserve de purger les listes des citoyens qui sont ou morts, ou domiciliés ailleurs, ou infirmes, ou analphabètes. Le principe de l'unanimité est conservé, sans délai de temps. Mais le projet de code criminel, présenté en juin 1801, n'est pas du goût des autorités chargées de l'examiner. L'atmosphère de réaction s'est généralisée.

En août 1802, un sénatus-consulte permet au Sénat de suspendre « pour cinq ans les fonctions de jurés dans les départements où cette mesure est nécessaire », de déclarer, « quand les circonstances l'exigent, des départements hors de la Constitution », d'annuler les jugements des tribunaux, lorsqu'ils sont attentatoires à la sûreté de l'État », ce que le Sénat fera abondamment y compris dans les causes individuelles. Lorsque les tribunaux sont appelés à donner leur avis sur le projet de code criminel (1803), le tribunal de cassation et le ministre de la justice désapprouvent profondément l'institution du jury, sans aller

44 SOLIMANO, Stefano. L'établissement de l'ordre juridique napoléonien : le rôle de Guy Jean-Baptiste Target. Forhistiuris, 2004. §15.

45 SOLIMANO, Stefano. Verso il Code Napoléon. Il progetto di Codice civile di Guy Jean Baptiste Target (1798-1799). Milan: Giuffré, 1998. p. 69 s.

46 BOUGLÉ, Claire. Le code d'instruction criminelle de 1808. In: HAUTEBERT, Joël; SOLEIL, Sylvain (eds.). La procédure et la construction de l'État en Europe $\left(X V I^{\mathrm{e}}-X I X^{\mathrm{e}}\right.$ siècles). Recueil de textes, présentés et commentés. Rennes: PUR, 2011. p. 604. 
jusqu'à réclamer son abrogation. La majorité des tribunaux d'appel, en revanche, demandent nettement sa suppression. Quant aux tribunaux criminels, une moitié environ se prononce pour le maintien du jury, une autre moitié contre ${ }^{47}$. Lorsque le Conseil d'État s'empare, à son tour, du projet (1804), c'est pour le critiquer. D'ailleurs, la première des quatorze questions à l'ordre du jour est : «L'institution du jury serat-elle ou non conservée ? ${ }^{48}$. Locré qui a rapporté les débats, raconte comment deux groupes de conseillers d'État s'étaient opposés : ceux qui, fidèles aux avantages du modèle anglais, défendent le projet de juin 1801 et cherchent à maintenir le double jury en s'appuyant sur l'héritage révolutionnaire et le fait qu'il était inscrit dans la constitution de l'an VIII ; ceux qui, plus en phase avec l'ambiance du moment, rejettent le double jury et réclament le retour à l'ordonnance criminelle de 1670. De nombreux facteurs jouent en faveur du second groupe : en 1804, la guerre a repris contre le Royaume-Uni ce qui explique le déclin de l'anglophilie ; Napoléon se montre ouvertement hostile au jury ; l'opinion publique exige, plus encore qu'en 1800, de la fermeté parce qu'elle est exaspérée par la criminalité, notamment celle des assassins, des voleurs, des bandes de chauffeurs qui, héritiers des criminels de grands chemins d'Ancien Régime, s'introduisent dans les fermes isolées, torturent les habitants en brûlant leurs pieds dans la cheminée pour connaître l'endroit où sont cachées les économies. Il faut encore évoquer l'idée partagée selon laquelle le jury serait mal adapté à la nation française à cause de l'esprit frivole, libre et critique des Français. Enfin et surtout, lors des débats, la mise en comparaison de l'idéalisme des Lumières avec les mécanismes éprouvés de l'ordonnance de 1670 tourne souvent en faveur de cette dernière. Elle va servir de modèle pour le contre-projet du Conseil d'État de 1804 qui, discuté de janvier à octobre 1808, sera adopté en tant que Code d'instruction criminelle en 1808.

47 ESMEIN, Adhémar. Histoire de la procédure criminelle en France. Paris: Larose \& Forcel, 1882. p. 485 s.; GAWELIK, Katy. Les sources de la codification pénale napoléonienne à la lumière des observations des tribunaux sur le projet de Code criminel de l'an IX. Thèse droit. Lille : Université de Lille 2, 2002.

48 PICARDI, Nicola; GIULIANI, Alessandro (eds.). I codici napoleonici, t. 2, Codice di instruzione criminale, 1808. Milan: Giuffré, 2002. p. XXXI. 
Qu'en est-il du jury dans ce code ${ }^{49}$ ? Le jury d'accusation, qui concentre toutes les critiques, disparaît. Les poursuites et l'instruction de l'affaire (organisée comme sous l'Ancien régime) sont confiées au procureur impérial et au juge d'instruction selon une procédure inquisitoire, secrète, non publique et non contradictoire. Lors de la clôture de l'instruction, le système est organisé en deux temps. Le juge d'instruction présente son rapport à la chambre du conseil, composée de lui-même et de magistrats du siège, en présence du procureur impérial. Il faut l'unanimité pour décharger l'inculpé de la plainte. Il suffit qu'un magistrat soit d'avis de poursuivre pour que le dossier soit transmis à la chambre d'accusation qui, sur le réquisitoire du procureur, décide ou non de rédiger un acte d'accusation et de renvoyer l'accusé devant la cour d'assises (le tribunal criminel, sous un autre nom). Le jury criminel de la cour d'assises, quant à lui, a été maintenu de justesse grâce à Treilhard qui, jusqu'au bout, aura plaidé en sa faveur et aura réussi à convaincre Napoléon de son utilité judiciaire et sociale. En réalité, à l'époque, une multitude d'affaires lui échappe au profit de juridictions criminelles qui, sous des noms divers, jugent sans jury. Au sein de la cour d'assises, le jury est composé de douze jurés, choisis au terme d'une sévère sélection. À partir des listes départementales, le préfet (le représentant du pouvoir exécutif dans le département) établit une liste de soixante jurés pour chaque assise. Puis, le président du tribunal réduit cette liste à trente-six. Enfin, le procureur général a le pouvoir de récuser jusqu'à douze noms, après que l'accusé a procédé, lui aussi, sans l'assistance de son avocat, jusqu'à douze récusations. À l'issue des débats publics et contradictoires, après qu'on a entendu la victime ou son avocat, puis le réquisitoire du procureur et les répliques de l'accusé ou de son avocat, le président est chargé de résumer l'affaire aux jurés. Ceux-ci, hors la présence des juges, délibèrent sur des questions dont le nombre a été réduit : 1) «l'accusé est-il coupable d'avoir commis tel crime avec toutes les circonstances expliquées dans l'acte d'accusation?»,2) «l'accusé a-t-il commis le crime avec telle circonstance particulière ? » et (au cas où l'accusé invoque un fait justificatif ou une circonstance atténuante) 3) «ce fait est-il constant? ». Les jurés, après avoir délibéré, donnent leurs réponses, l'un après l'autre,

49 BOUGLÉ. Le code (op. cit.). p. 606. 
au chef du jury (l'un des jurés tiré au sort). Le vote, désormais, est à la majorité simple. Si sept jurés sur douze estiment l'accusé coupable, il est condamné. Si sept jurés l'estiment innocent, il est acquitté. Le partage des voix tourne en faveur de l'accusé. L'appel reste impossible mais, quoique le Code indique " la décision du jury ne pourra jamais être soumise à aucun recours » (art. 350), la prudence conduit à inscrire dans le code la possibilité d'un renvoi devant un autre jury si, au prononcé du verdict, les juges déclarent unanimement que les jurés se sont trompés (art. 352), la possibilité d'une cassation pour des motifs de droit (art. 416 et s.) et d'une révision pour des motifs de fait, par exemple lorsqu'on rapporte la preuve d'un faux témoignage, la preuve que la victime, prétendument morte, est toujours en vie, ou la preuve que le crime a été commis par un autre que le condamné (art. 443 et s.). Ce système du Code d'instruction criminelle de 1808 clôt une décennie de débats à propos du jury et entre en vigueur en 1811.

\section{LA RESTAURATION ET LA SECONDE CONTROVERSE DOCTRINALE SUR LE JURY À L'ANGLAISE}

En 1814, puis en 1815, le système politique européen voulu par Napoléon s'effondre. Louis XVIII revient en France ; c'est la Restauration. Mais parce que Louis XVIII refuse de nouveaux soubresauts, il décide de conserver les institutions de Napoléon. Malgré quelques réformes, tous les codes sont maintenus, y compris le code d'instruction criminelle de 1808 et son système inquisitoire. Le roi choisit même de continuer à faire juger les affaires de nature politique par des juridictions d'exception, sans jury ${ }^{50}$. Cette nouvelle période s'ouvre donc sur une aspiration aux

50 MARTINAGE, Renée. Punir le crime. La répression judiciaire depuis le Code Pénal. Villeneuve d'Ascq: L'Espace Juridique, 1989; TORT, Olivier. La magistrature française face aux deux Restaurations (1814-1815). Revue d'Histoire du XIX ${ }^{\mathrm{e}}$ siècle, 2014. p. 93 s.; BOUGLÉ, Claire. La Cour de cassation et le code pénal de 1810. Le principe de légalité à l'épreuve de la jurisprudence (1811-1863). Paris: LGDJ, 2005; DONOVAN, James M. Juries and the Transformation of Criminal Justice in France in the Nineteenth \& Twentieth Century. Chapel Hill: UNCP, 2010; BERGER, Emmanuel; ROUSSEAUX, Xavier. Le jury criminel sous le Premier Empire. Origines et perspectives d'une tentative d'harmonisation juridique en Europe. In: ANTOINE, François; JESSENNE, Jean-Pierre; 
libertés publiques et individuelles, et sur une volonté de réhabiliter le jury en tant que symbole d'une justice d'équilibre. Ricard, Bérenger, Cottu, Rémusat, Comte, Legraverend, Heulhard de Montigny, Boyard, Guernon de Rainville, Oudart, Bavoux, Dupin, et d'autres, cherchent donc à mobiliser l'opinion publique et encourager des réformes audacieuses ${ }^{51}$. L'opinion du professeur Bavoux et de l'avocat Dupin est particulièrement intéressante car, en 1820, Bavoux a été traduit en justice après avoir été accusé d'avoir appelé ses étudiants à la désobéissance des lois, durant son cours de législation pénale ${ }^{52}$. Et parce que Bavoux a été défendu par Dupin en cour d'assises, les deux hommes critiquent le système de façon personnelle et passionnée.

Tout d'abord, chez ces auteurs, on ressent une même volonté de revanche à l'égard de Napoléon et de sa justice criminelle qui est présentée comme un instrument de dictature policière, administrative et répressive. En 1818, l'avocat général Bérenger, démissionnaire de la cour d'appel de Grenoble, stigmatise le Code d'instruction datant d'une période où «le gouvernement ne songeait à enchaîner alors que la liberté politique $»^{53}$. En 1819, le conseiller à la cour de cassation Oudart rappelle que le double jury criminel, quoique dénigré, a été sauvé par ceux « qui jugeaient que nous n'étions pas assez préparés à la perte du peu de libertés qui nous restaient, et qu'il n'était pas temps encore de renverser le jury $»^{54}$. En

JOURDAN, Annie; LEUWERS, Hervé (eds.). L'Empire napoléonien : une expérience européenne? Paris: Colin, 2014. p. 227 s.

${ }^{51}$ Sur ces auteurs : FALELAVAKI, Yannick. L'histoire d'une conversion. La doctrine française du XIX $X^{\mathrm{e}}$ siècle et le recours à la comparaison juridique. Thèse droit, Rennes : Université de 1, 2016; ARABEYRE, Patrick; HALPÉRIN, JeanLouis; KRYNEN, Jacques (eds.). Dictionnaire historique des juristes français, $X I I^{\mathrm{e}}-X X^{\mathrm{e}}$ siècles. Paris: PUF, 2007.

52 VENTRE-DENIS, Madeleine. La faculté de droit de Paris et la vie politique sous la Restauration. L'affaire Bavoux, Revue d'Histoire des facultés de Droit et de la Science Juridique, 1987. p. 44 s.; VIELFAURE, Pascal. L'art de convaincre dans les procès politiques en France sous la Restauration selon l'avocat Dupin aîné. In: Ars Persuasionis : entre doute et certitude. Berlin: Duncker \& Humblot, 2012. p. $89 \mathrm{~s}$.

53 BÉRENGER. De la justice criminelle en France, d'après les lois permanentes, les lois d'exception et les doctrines des tribunaux. Paris: L'Huillier, 1818. p. 4.

${ }^{54}$ OUDART. Essai sur l'organisation du jury de jugement et sur l'instruction criminelle. Paris: Delaunay, 1819. p. 3. 
1821, le professeur Bavoux déplore « le régime des décrets et des lois faites à huis clos », avec « tous les moyens qui n'étaient propres qu'à l'usurpation et à la tyrannie $»^{55}$.

Ensuite, chez ces auteurs, le jury fait l'objet de plaidoyers et de propositions. Cottu, conseiller à la cour d'appel de Paris, admet le manque d'entrain de la part des citoyens à l'égard de la fonction de juré, mais, souligne-t-il, « qu'a-t-on fait jusqu'ici pour mettre cette institution à l'honneur ? ${ }^{56}$. Le Graverend dément l'assertion devenue proverbiale selon laquelle le jury favorise l'impunité ${ }^{57}$. Bavoux estime que ses défauts n'étaient liés qu'à la disproportion des peines prévues par le code. Ce sont donc les lois pénales qu'il faut réformer. Dupin réclame le droit, pour les avocats, de participer à la récusation des jurés ${ }^{58}$. Certains accusent les magistrats d'avoir dénigré le jury pour obtenir son abolition et récupérer leurs anciens pouvoirs. D'autres se prononcent contre les juridictions d'exception qui jugent les affaires sensibles sans jury. Tous s'indignent contre le système de choix confié aux préfets, de sorte que les hommes désignés sont moins des jurés que des « commissaires » du gouvernement.

Enfin, et par opposition, tous ces auteurs se réfèrent à l'Angleterre et aux États-Unis pour, explique Oudart, « faire ressortir tous les vices du jury français ». « Il sera facile ensuite, en ajoutant à ces modèles ce qui serait jugé utile, et en y retranchant ce qu'on trouverait de défectueux, de former un système approprié à nos mœurs ». À chaque page, celui-ci défend l'institution originelle (anglaise) qui a été défigurée en France et conclut en proposant des solutions. Comte, après un long réquisitoire contre la procédure française, affirme que c'est la procédure par jurés qui a permis aux Anglais de résister aux lois tyranniques votées par le parlement. Pour exercer la tyrannie, explique-t-il, des lois cruelles ne suffisent pas, car il faut aussi une justice pour les appliquer : or, il n'y a rien à craindre de citoyens honnêtes qui sont appelés à juger de façon

55 BAVOUX. Leçons préliminaires sur le code pénal, ou examen de la législation criminelle. Paris: Bavoux, 1821. p. I. COTTU. Réflexions sur l'état actuel du jury, de la liberté individuelle et des prisons. Paris: Nicole, 1818. p. 84. nelle. Paris: Baudouin, 1821. p. 187-188. 
temporaire ${ }^{59}$. Toutefois, cette comparaison franco-britannique est assez différente de celle que les philosophes des Lumières alimentaient avant la Révolution. Elle s'appuie bientôt sur des observations menées sur place, en Angleterre, et sur une diversité de conclusions.

René Cottu, conseiller à la cour d'appel de Paris et secrétaire général de la société royale des prisons, a publié en 1818 un ouvrage critique : Réflexions sur l'état actuel du jury, de la liberté individuelle et des prisons. En 1820, il est envoyé en Angleterre par le gouvernement « pour y étudier le système et la marche du jury ». À son retour, il publie le résultat de ses discussions avec des parlementaires et des juges anglais et le fruit de ses observations lors des assises anglaises du circuit du Nord. Son ouvrage devient immédiatement un pivot de la réflexion sur le jury, aussi bien en France qu'à l'étranger où il est recensé dans les revues juridiques. Or, si l'ouvrage consiste à souligner l'excellence de la justice criminelle anglaise, il cherche également à montrer qu'elle est difficilement applicable en France. Après avoir longuement décrit et expliqué ce qu'il a vu, il en vient à affirmer que les hommes mobilisés par la justice anglaise, juges, shérifs, avocats et jurés, sont tous habités d'une même volonté : juger avec impartialité, respecter l'accusé, punir avec discernement ${ }^{60}$ :

« Il suit de tous ces détails [...] que les tribunaux anglais présentent un aspect d'impartialité et de douceur que les nôtres, il faut l'avouer, sont bien loin d'offrir aux yeux de l'étranger. Tout, en Angleterre, respire l'indulgence et la bonté ; le juge parait un bon père au milieu de sa famille occupé à juger un de ses enfants. Son aspect n'a rien d'effrayant. [...] Tout, chez nous, au contraire, paraît hostile contre l'accusé. Le ministère public le traite souvent avec une dureté, pour ne pas dire une cruauté, qui fait frémir les Anglais. Nos présidents eux-mêmes, loin de porter au prévenu l'intérêt qu'il aurait droit d'attendre au moins de l'impartialité de leur ministère, chargés par leurs fonctions de diriger les débats et d'établir l'accusation, deviennent trop souvent partie contre

59 COMTE. Des pouvoirs et des obligations des jurys par Sir Richard Philipps, traduit de l'anglais. Paris: Eymery, 1819. p. CIX-CX.

60 COTTU. De l'administration de la justice criminelle en Angleterre et de l'esprit du gouvernement anglais. Paris: Nicole, 1820. p. 109. 
l'accusé, et semblent quelquefois se faire moins un devoir qu'un honneur de le faire condamner. »

Dans le même temps, Cottu insiste sur le fait que le jury anglais est à l'image de la société anglaise. Elle est organisée autour d'une aristocratie provinciale et terrienne à la fois puissante et soucieuse de préserver les libertés publiques. C'est parmi cette aristocratie que l'on choisit les juges de paix et les shérifs dont l'une des missions est de nommer les membres du grand et du petit jury. Or, insiste Cottu, les sheriffs choisissent toujours les hommes les plus renommés du comté et l'on ne trouve donc jamais rien à redire à leur choix. Les listes de jurés sont composées sans suspicion, ni contestation. C'est un honneur d'avoir été choisi et, insiste Cottu, chaque Anglais connaît les devoirs de la charge et sait qu'un accusé ne peut être condamné que sur la décision de vingt-quatre de ses pairs : douze grands jurés au moins lors de la mise en accusation et douze petits jurés à l'unanimité lors du jugement. Là est le problème. Cottu, fidèle à Montesquieu, admire le système anglais et le met en relation étroite avec l'esprit public et les mœurs façonnées par l'histoire constitutionnelle et politique du pays. Or, déplore-t-il, en France, la Révolution a détruit l'aristocratie. Les bienfaits de la fin des privilèges et des préjugés se sont accompagnés de la ruine des familles titrées, ce qui rend impossible l'esprit public qui règne en Angleterre. L'auteur conclut en indiquant quelques réformes à faire en matière criminelle.

Quelques années plus tard, en 1828, Comte publie la seconde édition de sa traduction de l'ouvrage d'un shérif de Londres consacré au jury anglais. Cette édition, explique-t-il, est refondue non seulement parce que le système du jury criminel a été aménagé en France aussi bien qu'en Angleterre, mais aussi parce que l'auteur, Comte, a passé deux ans en Angleterre à observer la pratique des cours ${ }^{61}$. Il en revient encore plus convaincu de l'excellence du système anglais et de la nécessité de le prendre pour modèle. De l'autre côté de l'échiquier politique, on doit une autre approche à Joseph Rey, un magistrat et avocat, libéral ardent. Ayant participé au complot d'août 1820, il a été condamné à mort par

61 COMTE. Des pouvoirs et des obligations des jurys par Sir Richard Philipps, traduit de l'anglais. Paris: Rapilly, 1828. p. IV. 
contumace et s'est exilé en Angleterre. Il fonde sa réflexion pénale sur les Lumières radicales ${ }^{62}$. C'est lors de son séjour en Angleterre, qu'il a réuni les éléments de ses Institutions judiciaires de l'Angleterre comparées avec celles de la France, et de quelques autres États anciens et modernes. Gracié par Charles X en 1826, il rentre en France et publie, en 1828, l'ouvrage en deux volumes. S'il admire certains aspects du système britannique, il condamne fermement le droit et la justice du pays. Sans remettre en cause l'institution du jury qu'il juge préférable à tout autre système, Rey dresse une impressionnante liste de critiques. Tout d'abord, assure-t-il, les affaires criminelles sont loin d'être toutes jugées par un jury criminel ordinaire. Les jurys spéciaux menacent désormais de supplanter le jury ordinaire. Or, cette institution est « encore plus vicieuse qu'aucune partie du jury français $»^{63}$ : la liste des jurés est identique d'un procès à l'autre, ils sont nommés ou radiés de façon arbitraire par un officier de la cour, ils reçoivent un salaire considérable et sont, ainsi, soumis aux caprices du gouvernement, particulièrement pour les délits de presse. Ensuite, la formule « grand jury » est impropre car il n'est pas composé de jurés, mais des juges de paix assemblés pour délibérer sur les poursuites éventuelles, ce qui, en fait, rapproche l'institution de la chambre des mises en accusation française ${ }^{64}$. Enfin, il ne faut pas se méprendre sur la prétendue impartialité de la justice anglaise. Non seulement les juges influencent les jurés lorsqu'ils résument les débats et donnent leur avis sur la qualification criminelle et sur la peine, ou lorsqu'ils sermonnent les jurés pour les inviter à trancher dans tel ou tel sens, mais les jurés euxmêmes s'en remettent souvent au juge, soit par déférence, soit parce qu'ils sont indécis, soit parce qu'ils savent qu'une divergence entre leur verdict et l'opinion du juge sera une cause de pourvoi contre la décision du jury. Rey, comme d'autres étrangers qui ont assisté aux assises, témoigne de son étonnement à voir les jurés demeurer dans la salle du tribunal plutôt que de se retirer dans une salle à part, puis se tourner les uns vers les autres pour prendre leur décision, « ce qui est beaucoup plus expéditif

62 REGAD, Mathilde. Attaquer le droit par la philosophie. Le cas Joseph Rey. Paris: Campus Ouvert, 2016.

63 REY. Des institutions judiciaires de l'Angleterre comparées avec celles de la France, et de quelques autres États anciens et modernes, v. 2. Paris: Béchet, 1828. p. 48.

64 Ibid. p. 59. 
qu'en France $»^{65}$. Rey attire ainsi l'attention sur les fausses vérités du jury criminel anglais et force est de constater qu'il sera suivi par de nombreux auteurs français des années $1830^{66}$.

\section{Conclusions}

Cette étude des opinions doctrinales françaises montre comment le jury anglais est devenu, en France, un enjeu de controverses et de réformes. L'adoption du modèle anglais s'opère, en 1790-1791, dans l'enthousiasme. La pratique révèle à la fois le courage des jurés et la faillite de l'architecture pénale révolutionnaire. Une première controverse s'organise lorsque le Consulat pose franchement la question du maintien du jury. L'Empire modifie l'équilibre du système en supprimant le jury d'accusation et en se réservant le choix des jurés criminels. La chute de Napoléon suscite d'intenses plaidoyers en faveur du jury à l'anglaise. Mais les observations, sur place, de Cottu, Comte et Rey témoignent d'approches diverses, ce qui montre que l'imitation du modèle et la transplantation de l'institution n'ont rien d'évident. Plus globalement, l'analyse de cette littérature doctrinale met en lumière plusieurs éléments.

Tout d'abord, comme toujours en matière criminelle, on constate que les institutions chargées de lutter contre la criminalité et de juger les accusés font l'objet de débats très vifs. Montesquieu l'avait affirmé : comme il s'agit de disposer de la vie, de l'honneur ou des biens d'un homme ou d'une femme, accusés peut-être injustement d'avoir commis un crime, rien ne saurait être plus sérieux. Certes. Mais comme le droit criminel vise aussi à défendre l'ordre public, la vie, l'honneur et les biens des victimes, où placer le point d'équilibre? Les auteurs, philosophes, hommes politiques et praticiens s'emparent du sujet, débattent et publient selon des cycles continuels, car l'équilibre reste précaire et dépend d'un nombre variable de facteurs.

Ibid. p. 365-366.

SOLEIL, Sylvain. La concurrence entre droit français et droit anglais. Antagonismes et systématisations vus de France (1825-1850). Revue de Droit de l'Université de Sherbrooke, à paraître. 
Ensuite, comme toujours en matière criminelle, on ressent cette dialectique constante entre les principes et la pratique, entre la volonté d'établir une justice criminelle réglée, stable, équilibrée, et les chiffres implacables de la criminalité. En ce sens, l'institution du jury fonctionne tantôt, en 1790 ou en 1818-1820, comme le symbole d'un édifice judiciaire humain et harmonieux, tantôt, en 1795-1808 ou sous la plume de Rey, comme une technique qu'il s'agit d'examiner à la lumière de l'expérience, de critiquer, voire d'abroger.

Le thème dévoile encore plusieurs façons d'envisager la comparaison des droits : il y a les auteurs qui se servent du jury anglais comme d'un argument contre le système français sans avoir vérifié leurs sources ; il y a ceux qui cherchent vraiment à comprendre l'intelligence du système pour le comparer avec le système français ; il y a enfin ceux qui réutilisent les données des deux premiers types d'auteurs pour nourrir leurs grandes doctrines pénales. On retrouve ici l'approche du droit comparé par les mobiles : comparer pour chercher des modèles à imiter ou à rejeter, comparer pour comprendre scientifiquement un droit étranger, comparer pour alimenter des théories ${ }^{67}$.

Enfin, ces premières controverses permettent de comprendre la différence entre la perspective britannique et nord-américaine, où l'institution du jury a toujours été considérée comme le refuge des libertés publiques et n'a jamais été menacée malgré les critiques qui lui ont été adressées (et qui demeurent aujourd'hui encore : mode de désignation et de récusation, méthode de profilage du jury idéal, impartialité des jurés, etc.), et la perspective française, où l'hypothèse de l'abolition du système était crédible dès la transplantation originelle et va régulièrement ressurgir au XIX ${ }^{\mathrm{e}}$ siècle : le jury, en France, ne fait pas l'objet du même attachement parce que l'histoire en a fait une institution liée à la Révolution française et ses excès, parce que les controverses et les ajustements législatifs permanents en ont fait une institution discutable, précaire, modulable. En 1890, Gabriel Tarde ne fait pas scandale en en parlant comme d'une institution « aussi inefficace qu'inintelligente ». Plus récemment, la réforme

67 PADOA-SCHIOPPA, Antonio (ed.). La comparazione giuridica tra Ottocento e Novecento. In memoria di Mario Rotondi. Milan: Ist. Lombardo, 2001; SOLEIL, Sylvain. Por que comparar os direitos no século XIX. Revista da Faculdade de Direito da UFRGS, 2020. p. 1-48. 
du 15 juin 2000 consistant à soumettre les verdicts des cours d'assises au double degré de juridiction (en contradiction flagrante avec le principe suivant lequel c'est le peuple qui juge à travers les jurés) ou celle du 23 mars 2019 qui crée des cours criminelles composées exclusivement de magistrats professionnels et chargées de juger les crimes punis de quinze ou vingt ans de réclusion criminelle, ont été faites sans émoi dans l'opinion publique. Le modèle était anglais, mais la greffe a été faite dans un organisme qui n'est pas l'Angleterre.

\section{RÉFÉRENCES}

AGOSTINI, Éric. La circulation des modèles juridiques. Revue Internationale de Droit Comparé, 1990. p. 461 s.

ALLEN, Robert. Les tribunaux criminels sous la révolution et l'Empire (1792-1811). Rennes: PUR, 2005. \$16 s. See: https://books.openedition.org/pur/8128.

ANONYME. Aux rédacteurs de la Revue, La Revue Philosophique, Littéraire et Politique, 1804-1805. p. 12 s.

ARABEYRE, Patrick; HALPÉRIN, Jean-Louis; KRYNEN, Jacques (eds.). Dictionnaire historique des juristes français, $X I I^{\mathrm{e}}-X X^{\mathrm{e}}$ siècles. Paris : Presses universitaires de France, 2007.

ASTAING, Antoine. Droits et garanties de l'accusé dans le procès criminel d'Ancien Régime (XVI $-X V I I I^{e}$ siècle). Audace et pusillanimité de la doctrine pénale française. Aix-en-Provence: PUAM, 1999.

BAKER, John H. An Introduction to English Legal History. London: Butterworths, 2000.

BAVOUX. Leçons préliminaires sur le code pénal, ou examen de la législation criminelle. Paris: Bavoux, 1821.

BEATTIE, John. Crime and the Courts in England, 1660-1800. Oxford: Clarendon Press, 1986.

BÉRENGER. De la justice criminelle en France, d'après les lois permanentes, les lois d'exception et les doctrines des tribunaux. Paris: L'Huillier, 1818.

BERGER, Emmanuel. Ordre public et poursuites criminelles sous le Directoire (1795-1799). Annales Historiques de la Révolution Française, 2007. p. 146 s. 
BERGER, Emmanuel. La justice pénale sous la Révolution. Les enjeux d'un modèle judiciaire libéral. Rennes: PUR, 2008.

BERGER, Emmanuel. The Criminal Jury in England and France in the late $18^{\text {th }}$ Century. Historiographical Issues and Research Perspectives of Popular Justice. In: DELIVRÉ, Emilie; BERGER, Emmanuel (eds.). Popular Justice in Europe (18 ${ }^{\text {th }}$ $19^{\text {th }}$ Centuries). Berlin: Duncker \& Humblot. 2014. p. 71 s.

BERGER, Emmanuel; ROUSSEAUX, Xavier. Le jury criminel sous le Premier Empire. Origines et perspectives d'une tentative d'harmonisation juridique en Europe. In: ANTOINE, François; JESSENNE, Jean-Pierre; JOURDAN, Annie; LEUWERS, Hervé (eds.). L'Empire napoléonien: une expérience européenne? Paris: Colin, 2014. p. 227 s.

BLOT-MACCAGNAN, Stéphanie. Procédure criminelle et défense de l'accusé à la fin de l'Ancien Régime. Rennes: PUR, 2010.

BOUGLÉ, Claire. La Cour de cassation et le code pénal de 1810. Le principe de légalité à l'épreuve de la jurisprudence (1811-1863). Paris: LGDJ, 2005.

BOUGLÉ, Claire. Le code d'instruction criminelle de 1808, In: HAUTEBERT, Joël; SOLEIL, Sylvain (eds.). La procédure et la construction de l'État en Europe (XVI$X I X^{\mathrm{e}}$ siècles). Recueil de textes, présentés et commentés. Rennes: PUR, 2011. p. $600 \mathrm{~s}$.

BOULANT, Antoine. Le tribunal révolutionnaire. Punir les ennemis du peuple. Paris: Perrin, 2018.

BOURGUIGNON. Mémoire sur les moyens de perfectionner en France l'institution du jury. Paris: Maradan, 1802.

BOURGUIGNON. Deuxième mémoire sur l'institution du jury. Paris: Patris, 1804.

BREXON. Développement de la théorie des lois criminelles. Paris: Garnery, 1802.

BRISSOT. Bibliothèque philosophique... 10 vols. Paris: Desauges, 1782-1785.

BROWN, Howard G. Ending the French Revolution: Violence, Justice, and Repression from the Terror to Napoleon. Charlottesville: UVP, 2006.

CAIRNS, John W. Watson, Walton, and the History of Legal Transplants. Georgia Journal of International and Comparative Law, 2014. p. 637 s.

CANARD. Moyens de perfectionner le jury. Moulins: Vidalin, 1802.

CARBASSE, Jean-Marie. État autoritaire et justice répressive: l'évolution de la législation pénale de 1789 au Code de 1810. In: All'ombra dell'aquila imperiale. Rome: Archivi di Stato, 1994. p.325 s. 
CARBASSE, Jean-Marie. Histoire du droit pénal et de la justice criminelle. Paris: PUF, 2000.

CARBONNIÈRES, Louis (de). Les destinées du jury français et du jury anglais (XVIII ${ }^{\mathrm{e}}-\mathrm{XIX}{ }^{\mathrm{e}}$ siècles). Les Cahiers de la Justice, Paris, 2012. p. 83 s.

CARBONNIÈRES, Louis (de). Le comte de Lauraguais, anglomane isolé, face au jury révolutionnaire. Revue Historique de Droit Français et Etranger, 2013. p. 283 s.

COMTE. Des pouvoirs et des obligations des jurys par Sir Richard Philipps, traduit de l'anglais. Paris: Eymery, 1819.

COTTU. Réflexions sur l'état actuel du jury, de la liberté individuelle et des prisons. Paris: Nicole, 1818.

COTTU. De l'administration de la justice criminelle en Angleterre et de l'esprit du gouvernement anglais. Paris: Nicole, 1820.

CRÉPIN, Marie-Yvonne. Les Thermidoriens et la reconstruction juridique. In: $L a$ Vendée. Après la Terreur, la reconstruction. Paris: Perrin, 1997. p.191 s.

CRÉPIN, Marie-Yvonne. Ordonnance criminelle dite de Saint-Germain-en-Laye, août 1670. In: HAUTEBERT, Joël; SOLEIL, Sylvain (eds.). La procédure et la construction de l'État en Europe (XVI ${ }^{\mathrm{e}}-X I X^{\mathrm{e}}$ siècles). Recueil de textes, présentés et commentés. Rennes: PUR, 2011. p. 476 s.

DEBAUVE, Jean-Louis. La justice révolutionnaire dans le Morbihan (1790-1795). Paris: Debauve, 1965.

DONOVAN, James M. Juries and the transformation of Criminal Justice in France in the Nineteenth \& Twentieth Century. Chapel Hill: UNCP, 2010.

DRUGEON, Laurent. Les sources du droit procédural révolutionnaire: une voie française ? In: HAUTEBERT, Joël; SOLEIL, Sylvain (eds.). Modèles français, enjeux politiques et élaboration des grands textes de procédure en Europe. v. 2. Paris: EJT, 2012. p.13 s.

DUPIN. Observations sur plusieurs points importants de notre législation criminelle. Paris: Baudouin, 1821.

ESMEIN, Adhémar. Histoire de la procédure criminelle en France. Paris: Larose \& Forcel, 1882.

FALELAVAKI, Yannick. L'histoire d'une conversion. La doctrine française du $X I X^{\mathrm{e}}$ siècle et le recours à la comparaison juridique. Thèse droit, Rennes : Université de Rennes 1, 2016. 
GACH. Des vices de l'institution du jury en France. Paris: Petit, 1804.

GAUDEMET, Jean. Les transferts de droit. Année sociologique, 1976. p. 29 s.

GAWELIK, Katy. Les sources de la codification pénale napoléonienne à la lumière des observations des tribunaux sur le projet de Code criminel de l'an IX. Thèse droit, Lille : Université de Lille 2, 2002.

GRANGER. Idée des abus existants dans l'ordre de procéder en matière criminelle. Paris: Guerbart, 1800.

HALPÉRIN, Jean-Louis. Haro sur les hommes de loi. Droits, 1993. p. 55 s.

HYNT, Lyne. Politics, Culture, and Class in the French Revolution. Berkeley: UCP, 1984.

KING, Peter. Crime, Justice and Discretion in England, 1740-1820. Oxford: OUP, 2000.

LAFON, Jacqueline-Lucienne. La Révolution française face au système judiciaire d'Ancien Régime. Genève : Droz, 2001.

LANDRON, Gilles. À propos de la "commisération abusive" du jury criminel de la Révolution. Histoire de la Justice, 1994. p. 73 s.

LEGRAND, Pierre. The impossibility of "Legal Transplants”. Maastricht Journal of European and Comparative Law, 1997. p. 111 s.

LEGRAVEREND. Observations sur le jury en France. Paris: Delaunay, 1819.

MARI, Éric (de). La mise hors de la loi sous la Révolution française (1793-an III). Paris: LGDJ, 2015.

MARTINAGE, Renée. Punir le crime. La répression judiciaire depuis le Code Pénal. Villeneuve d'Ascq: L'Espace Juridique, 1989.

MARTUCCI, Roberto. La Costituante ed il problema penale in Francia (1789-1791), 1. Alle Origine dei processo accusatorio: I decreti Beaumetz. Milan: Giuffrè, 1984.

MAYENBURG, David (von); COLLIN, Peter; DECOCK, Wim; GROTKAMP, Nadine; SEENLENTAG, Anna (eds.). Geschichte der Konfliktregulierung in Europa - Ein Handbuch. Berlin: Springer, 2021.

MÉNY, Yves (ed.). Les politiques du mimétisme institutionnel, La greffe et le rejet. Paris: L'Harmattan, 1993.

OUDART. Essai sur l'organisation du jury de jugement et sur l'instruction criminelle. Paris: Delaunay, 1819. 
PADOA-SCHIOPPA, Antonio (ed.). The Trial Jury in England, France, Germany, 1700-1900. Berlin: Duncker \& Humblot, 1987.

PADOA-SCHIOPPA, Antonio (ed.). La comparazione giuridica tra Ottocento e Novecento. In memoria di Mario Rotondi. Milan: Ist. Lombardo, 2001.

PASSANO, Mario (da). Emendare o intimidire? La codificazione del diritto penale in Francia e in Italia durante la Rivoluzione e l'Impero. Torino: Giappichelli, 2000.

PICARDI, Nicola; GIULIANI, Alessandro (eds.). I codici napoleonici, t. 2, Codice di instruzione criminale, 1808. Milan: Giuffré, 2002.

PORCHER. L'excellence de l'institution du jury, Orléans: Guyot, 1804.

RAY, Jean. La Révolution française et la pensée juridique: l'idée de règne de la loi. Revue Philosophique, 1939.

REGAD, Mathilde. Attaquer le droit par la philosophie. Le cas Joseph Rey. Paris: Campus Ouvert, 2016.

REY. Des institutions judiciaires de l'Angleterre comparées avec celles de la France, et de quelques autres États anciens et modernes. Paris: Béchet, 1828, 2 vol.

RODIÈRE, René. Approche d'un phénomène: les migrations de systèmes juridiques. In: Mélanges Marty. Toulouse: PUT, 1978. p. 947 s.

ROUSSEAUX, Xavier. Politique judiciaire, criminalisation et répression. La révolution des juridictions criminelles 1792-1800). In: MARTIN, Jean-Claude (ed.). La Révolution à l'œuvre. Perspectives actuelles dans l'histoire de la Révolution française. Rennes: PUR, 2005. See: https://books.openedition.org/pur/16029.

SCHNAPPER, Bernard. L'activité du tribunal criminel de la Vienne, 1792-1800. In: La Révolution et l'ordre juridique privé: rationalité ou scandale? V. 2, Paris: PUF, 1988. p.623 s.

SCHNAPPER, Bernard. Le jury criminel. In: BOUCHER, Paul (ed.). Une Autre Justice, 1789-1799. Paris: Fayard, 1989. p. 170 s.

SMITH, Bruce P. The myth of private prosecution in England, 1750-1850. In: DUBBER Markus D.; FARMER Lindsay (eds.). Modern Histories of Crime and Punishment. Stanford: SUP, 2007. p. 151 s.

SOLEIL, Sylvain. Lex Imperat: Creation and Exportation of the French Model of the Legality Principle (18th-19th C.). In: MARTYN, Georges; DUBBER Marcus D.; PIHLAJAMÄKI Heikki (eds.). From the Judge's Arbitrium to the Legality 
Principle - Legislation as a Source of Law in Criminal Trials. Berlin: Duncker \& Humblot, 2013. p. $145 \mathrm{~s}$.

SOLEIL, Sylvain. Por que comparar os direitos no século XIX. Revista da Faculdade de Direito da UFRGS, 2020. p. 1 s.

SOLEIL, Sylvain. La concurrence entre droit français et droit anglais. Antagonismes et systématisations vus de France (1825-1850). Revue de Droit de l'Université de Sherbrooke, à paraître.

SOLIMANO, Stefano. L'établissement de l'ordre juridique napoléonien: le rôle de Guy Jean-Baptiste Target. Forhistiuris, 2004. See: https://forhistiur. net/2004-02-solimano.

SOLIMANO, Stefano. Verso il Code Napoléon. Il progetto di Codice civile di Guy Jean Baptiste Target (1798-1799). Milan: Giuffré, 1998.

SWIECICKA, Paulina. "Connectiones inter iura variorum observamus...”. En recherchant l'explication de la notion d'acculturation juridique. In: COPPEIN, Bart; STEVENS, Fred; WAELKENS, Laurent (eds.). Modernisme, Tradition et acculturation juridique. Bruxelles: Iuris Scripta Historica. 2011, p. 7 s.

TARDE, Gabriel. Les lois de l'imitation (1890). Paris: 1895.

TILLET Edouard. La constitution anglaise, un modèle politique et institutionnel dans la France des Lumières. Aix-Marseille: PUAM, 2001.

TIYAL, Samyr. Jacques-Pierre Brissot de Warville, genèse d'une pensée (1770-1789). Lille: ANRT, 1997.

TORT, Olivier. La magistrature française face aux deux Restaurations (1814-1815). Revue d'Histoire du XIX ${ }^{\mathrm{e}}$ siècle, 2014. p. 93 s.

VENTRE-DENIS, Madeleine. La faculté de droit de Paris et la vie politique sous la Restauration. L'affaire Bavoux. Revue d'Histoire des facultés de Droit et de la Science Juridique, 1987. p. 44 s.

VIELFAURE, Pascal. L'art de convaincre dans les procès politiques en France sous la Restauration selon l'avocat Dupin aîné. In: Ars Persuasionis: entre doute et certitude. Berlin: Duncker \& Humblot, 2012. p. 89 s.

WATSON, Albert. Legal Transplants: An Approach to Comparative Law. Édimbourg: Scottish Academic Press, 1974.

WESSEL, Albert. Thèse sur le jury criminel. Genève: Jarrys, 1849. 


\section{Additional information and author's declarations (scientific integrity)}

Conflict of interest declaration: the author confirms that there are no conflicts of interest in conducting this research and writing this article.

Declaration of authorship: all and only researchers who comply the authorship requirements of this article are listed as authors; all coauthors are fully responsible for this work in its entirety.

Declaration of originality: the author assures that the text here published has not been previously published in any other resource and that future republication will only take place with the express indication of the reference of this original publication; he also attests that there is no third party plagiarism or self-plagiarism.

\section{Editorial process dates}

(http://www.ibraspp.com.br/revista/index.php/RBDPP/about/editorialPolicies)

- Submission: $13 / 04 / 2021$

- Desk review and plagiarism check: 15/04/2021

- Review 1: 26/04/2021

- Review 2: 13/05/2021

- Preliminary editorial decision: $13 / 06 / 2021$

- Correction round return: $24 / 06 / 2021$

- Final editorial decision: 10/07/2021

\section{Editorial team}

- Editor-in-chief: 1 (VGV)

- Associated-editor: 1 (RS e GM)

- Reviewers: 2 


\section{HOW TO CITE (ABNT BRAZIL):}

SOLEIL, Sylvain. Le jury criminel en procès. Les opinions doctrinales des auteurs français (1750-1830). Revista Brasileira de Direito Processual Penal, vol. 7, n. 2, p. 763-800, mai./ago. 2021. https://doi.org/10.22197/rbdpp.v7i2.595 